\title{
A Collaborative Multimedia Annotation Tool for Enhancing Knowledge Sharing in CSCL
}

Stephen J.H. Yang ${ }^{a^{*}}$, Jia Zhang ${ }^{b}$, Addison Y.S Su ${ }^{\text {c }}$, Jeffrey J.P. Tsai ${ }^{\mathrm{d}}$

${ }^{a, c}$ National Central University, Taiwan; ${ }^{b}$ Northern Illinois University, USA; ${ }^{d}$ University of Illinois at Chicago, USA

Knowledge sharing in computer supported collaborative learning (CSCL) requires intensive social interactions among participants, typically in the form of annotations. An annotation refers to an explicit expression of knowledge that is attached to a document to reveal the conceptual meanings of an annotator's implicit thoughts. In this research, we develop a Semantic Web and Web services-supported multimedia tool to facilitate collaborative annotation sharing in the context of CSCL. Our experimental results demonstrate that our tool can facilitate knowledge sharing and improve participating students' reading comprehension: it helps participants raise good questions and provide proper answers through the practice of reading, commenting, reviewing, and discussion.

Keywords: collaboration; multimedia annotation; knowledge sharing; computer supported collaborative learning

\section{Introduction}

The advancement of computer science in recent years paves a way toward a new style of cooperative learning. Computer supported collaboration techniques allow students to study in a virtual team without physically sitting at a common place (Chung, Severance et al. 2003). Computer-supported collaborative learning (CSCL) was thus coined in 1996 (Koschmann 1996) to refer to a computer and network-supported collaborative learning environment for students to study cooperatively to acquire and share knowledge.

A CSCL-oriented software system is normally called a collaborative learning environment (CLE) (Littleton \& Häkkinen 1999; Komis, Avouris et al. 2002; Kreijns, Kirschner et al. 2005; Kreijns, Kirschner et al. 2007), focusing on using computer technologies to enhance interactions, communication, coordination, and interactivity within a learning group (Zurita \& Nussbaum 2004b). Among others, the performance of a CLE is fundamentally decided by how effectively and efficiently it supports knowledge sharing in a formed learning group. Although there is no common definition for the term knowledge sharing, it is widely recognized that it involves collaborative efforts by knowledge consumers and knowledge producers to exchange 
ideas and experiences. Annotation-based collaboration is one important way to facilitate knowledge sharing.

The world standard organization W3C defines annotations as comments, notes, explanations, or other types of external remarks, which are attached to a document or a selected part of the document (Brickley 2004). In general, annotations may help participants in four significant aspects: attention, organization, indexing, and discussion. Annotations may catch participants' attention thus help them focus on annotated concepts or subjects. In addition, annotations could help participants construct their own knowledge and concepts. Furthermore, annotations could help participants bookmark annotated areas and use them as indexes or reminders for later references. Moreover, embedded annotations may help participants discuss and review others' ideas and thoughts. Researchers have discovered that materials recorded as annotations are more likely to be recalled compared to those not recorded (Aiken, Thomas et al. 1975).

In a CLE, by reading and making annotations, participants collaboratively explore and exploit valuable ideas and knowledge. Experienced participants (e.g., experts and teachers) may also provide their annotations to help novices understand materials. Therefore, annotations can be considered as valuable knowledge in a CLE and thus require effective management. In recent years, a number of shared annotation provisioning systems have been developed (Russell 2005), represented by W3C's Annotea (W3C) and Microsoft's OneNote (Microsoft 2007). Meanwhile, many researchers focus on standardizing annotation definition and description. The resulting formats have been proposed as metadata (Gašević, Jovanović et al. 2007), such as Dublin Core (Initiative), Learning Object Metadata (LOM), and Shareable Content Object Reference Model (SCORM).

Our research aims to apply the latest Semantic Web and Web services technologies to build a Web-based social software tool for facilitating knowledge sharing through multimedia annotation management in a CSCL group. In our previous work (Yang, Chen et al. 2004), we develop a personalized annotation management system (PAMS) 1.0 as our kernel system, which provides a cluster of system-level facilities (i.e., annotation creation, editing, and retrieval) for users to annotate a shared document at various granularities. In this paper, we report how we apply the Semantic Web and Web services technologies to build a Web-based PAMS 2.0 featured with extensibility and interoperability. We explain how we leverage PAMS 2.0 to facilitate knowledge sharing in a CSCL context. We have also designed and conducted a series of experiments to evaluate the performance of our PAMS 2.0. Specifically, we examine whether PAMS could help participants raise good questions and provide answers through the practice of reading, commenting, reviewing, and discussion. Our experimental 
results demonstrate that multimedia annotation-based collaboration fa cilitates knowledge sharing and significantly improves participating students' reading comprehension.

The remainder of the paper is organized as follows. In Section 2, we discuss related work. In Section 3 and 4, we discuss how we built PAMS 2.0 and how PAMS can be used to facilitate collaborative annotation management, respectively. In Section 5 , we present our experiments and discuss our findings. In Section 6, we make conclusions.

\section{Related work}

\subsection{Knowledge sharing in CSCL}

A number of CSCL researchers focus on how to use computer technologies to enhance interaction, communication, coordination, negotiation, and interactivity within a learning group. Kollias et al. (Kollias, Mamalougos et al. 2005) study, from a teacher's perspective, how Web-based CSCL can complement and improve classroom study. Kreijns et al. (2007) build powerful learning environments such as Conversant Media and meta-cognition. Manlove et al. (Manlove, Lazonder et al. 2006) conclude from a case study that CSCL tools promote student learning through scientific inquiry learning.

It has been demonstrated that social relationships have impact on knowledge acquisition in a collaboration mode (Fischer, Bruhn et al. 2002). Our previous work (Yang, Zhang et al. 2007; Yang \& Chen 2008) proposes a social network-based Peer-to-Peer search method for identifying proper group study collaborators, who possess related knowledge and are willing to share corresponding knowledge. In contrast, this research builds a tool to facilitate knowledge sharing after a collaborative learning group is formed.

A term "community of practice" $(\mathrm{CoP})$ is coined from social science to refer to a group of people who share a common interest and study how they learn and deepen their knowledge and expertise through regular interactions (Wenger, McDermott et al. 2002; Alani, Dasmahapatra et al. 2003). Guldberg \& Pilkington (Guldberg \& Pilkington 2006) report that knowledge sharing is a motivation for most participants to use CoPs. Many CoP studies have elaborated on how to manage messages and documents posted in discussion forums.

Some researchers track and analyze interaction patterns to guide collaborative learning. Avouris et al. (Avouris, Komis et al. 2004) design an environment for monitoring and examining group learning patterns from two aspects: activity analysis and collaboration analysis. Collaboration feedbacks are also used to enhance 
collaborative e-learning (Zumbach, Hillers et al. 2003). Bravo et al. (Bravo, Redondo et al. 2006) build a learner-centered synchronous CSCL environment for students to study design and report a case study. Three groups of researchers, Lundin et al. (Lundin \& Magnusson 2003), Zurita et al. (Zurita \& Nussbaum 2004a), and Nicol et al. (Nicol \& MacLeod 2005), each examines some Web-based case studies in a mobile environment using handheld devices. Through the development of two Web-based collaborative learning environments, Rubens et al. (Rubens, Emans et al. 2005) summarize a set of pedagogical principles.

In summary, collaborative and cooperative learning approaches such as learning together, student team learning, group investigation, jigsaw, and dyadic cooperative learning, can be applied to many instructional scenarios (Hwang, Wang et al. 2007). Based on the pedagogy theories of collaborative and cooperative learning, the experiment class in our research was organized by group collaborative learning activities and an annotation system was provided to study whether multimedia annotation-based collaboration can facilitate knowledge sharing in a collaborative learning group.

\subsection{Multimedia annotation and annotation management}

Aiken, Thomas and Schennum (Aiken, Thomas et al. 1975) find that materials recorded in a learner's notes are twice as likely to be recalled as those not recorded. Their experiments also show that the team taking notes shows better organization and verbal capability in tests. Sannomiya et al. (Sannomiva, Amagasa et al. 2001) identify the core functions that annotation sharing should comprise: writing annotations, browsing annotations, and providing feedbacks.

Cater et al. (Carter, Churchill et al. 2004) study the effectiveness of multimedia annotation by providing methods for people to post and acquire materials. They modify and annotate previously posted annotations to create publicly observable threads. Hwang et al. (Hwang, Wang et al. 2007) propose a multimedia annotation system oriented to Web-based learning. They develop Web-based tools for creating and sharing annotations, and conduct experiments at college level students. Their experimental results show the benefits of using annotation systems on both individual learning and group learning. However, their systems lack a direct relationship between semantic technology and multimedia annotation, which is important for Web-based annotation query and management.

W3C has proposed a Web-based shared annotation system based on a general-purpose open Resource Definition Framework (RDF) infrastructure, called Annotea (W3C). Users use a Web-based Amaya editor (W3C) to browse Web content and make annotations in Annotea. The annotations can be stored either on annotation 
severs or on local machines. To associate annotations with annotated objects, Annotea utilizes the XPointer technology to insert annotation positions into the annotated document in XML. However, due to the overhead of maintaining linking relations, XPointer is only suitable for static documents; it does not work well with frequently changed documents. The reason is XPointer requires that an XML document labels the $i d$ attribute on every element that may be associated with annotations. Furthermore, XPointer can only apply to XML documents; therefore, Annotea only supports XML-based annotation. In contrast, our proposed tool is not limited to XML documents, and our tool does not require a document to be labeled before it can be annotated.

Microsoft has published OneNote that is a personalizable annotation tool. Users can choose a pen, a keyboard or voice to input annotations. However, notes can only be shared through e-mails. As a result, the usage of the tool is largely limited. IBM's Lotus Notes (IBM) allows discussions on a document. However, all comments can only be attached to the entire document, instead of to its comprising sections. CommonSpace (Surman \& Wershler-Henr 2002) allows interactions between teachers and students. A student can write annotations on a document; a teacher can comment on the annotations; and the student can further revise the annotations based on the teacher's comments. Meanwhile, the teacher can view all the annotations and comments attached to the document. However, the system is a stand-alone software product, not designed for Internet-based collaborations. In contrast with these annotation approaches that bind annotations directly to their corresponding annotated areas, our PAMS separates the storage of annotations from their corresponding annotated areas so that the annotations can be managed and retrieved independently. This feature is critical when multiple groups of people annotate on the same shared document simultaneously, which is an important requirement as a Web-based annotation system.

\section{Personalized annotation management system (PAMS) 2.0}

In a CSCL context, we define an annotation as an explicit expression of knowledge of a participant (annotator) in the form of a comment (note) or a question, which reveals conceptual meanings of the annotator's implicit thoughts. In this research, we call people's annotations as "personalized annotations" in the sense that each annotation is made by one individual to represent his/her personal understanding and thoughts. Therefore, an annotation is typically featured by the annotator's personal characteristics and preferences.

Our previous work yields a personalized annotation management system (PAMS) 1.0 (Yang, Chen et al. 2004). Upon a shared document, users can not only create and 
edit annotations, but also query about related annotations. In this project, we intend to upgrade PAMS and build a Web-based collaborative annotation environment.

\subsection{Annotation modeling}

A Web-based annotation management system has to take into consideration its potentially much broader user base and much more complex usage scenarios. For example, a document may be simultaneously studied and annotated by multiple learning groups. Therefore, it may be desired that all annotation activities be associated with corresponding learning groups. To enable effective and efficient annotation management, our strategy is to model annotation-related concepts using the Semantic Web technique. Specially, we introduce a concept of annotator group to delimit the domain of a learning group, and we decouple annotations from annotated objects (portion) for flexibility.

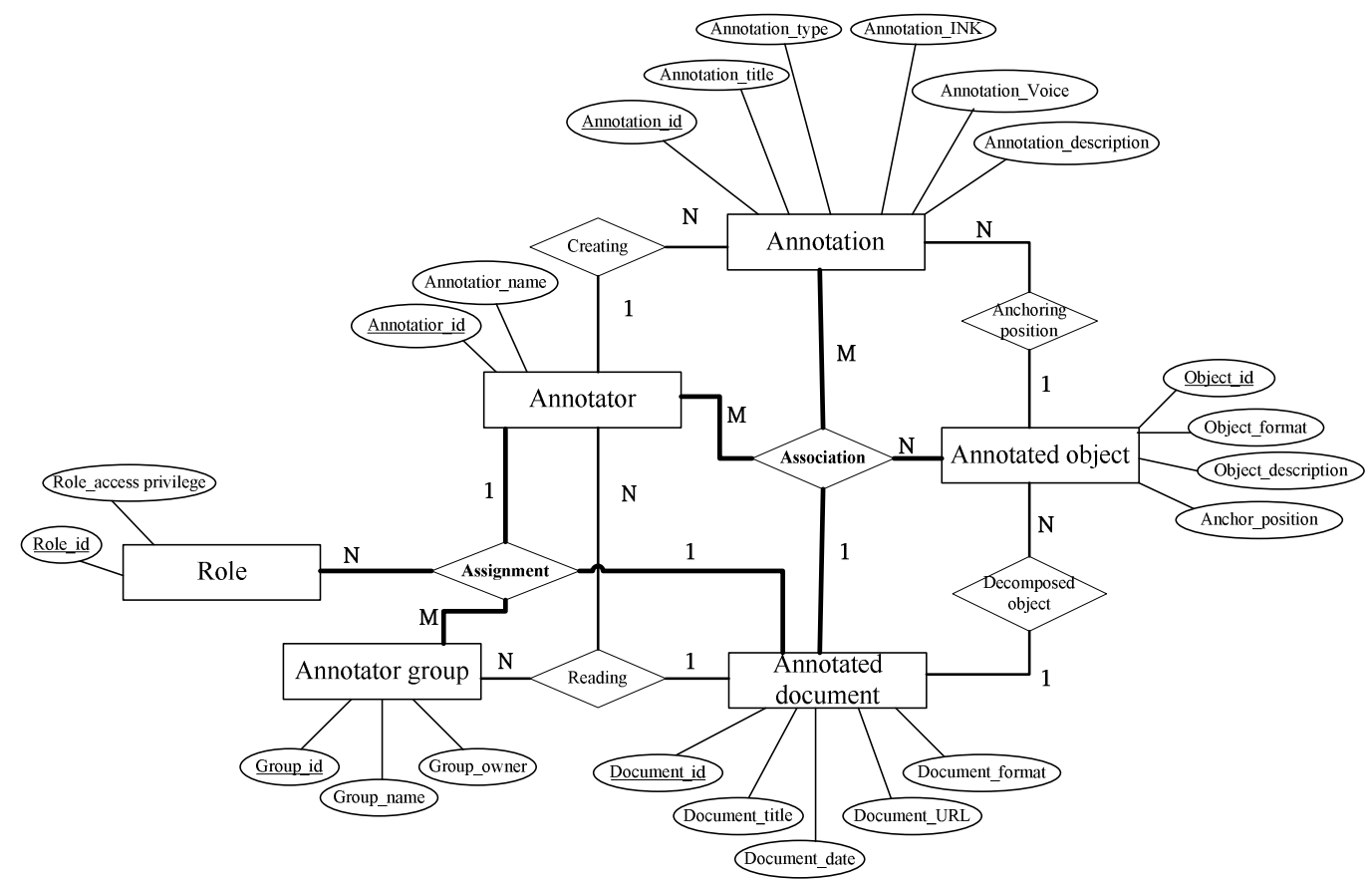

Figure 1. A PAMS 2.0 annotation Entity-Relation model.

We identify six conceptual elements regarding annotations. Figure 1 shows our annotation model as an ontological Entity-Relation model to illustrate these conceptual elements. A document with annotations is referred to an annotated document. Annotations can be created by any annotator upon any part (area) in the document, such as a word, a sentence, a section, or even the entire document. An area associated with annotations is called an annotated object. Annotated documents may allow role-based access control (e.g., an annotator may have read and write privileges 
to an annotated document). All annotators having the same access privilege (role) to a particular annotated document form an annotator group. The relationship between a specific annotation with its corresponding annotated object, annotated document, and annotator is recorded in an association. The relationship between a specific annotator with its corresponding role, annotated document, and annotator group is recorded in an assignment.

\subsection{WSRF-based annotation modeling}

We consider exploring different techniques to model this annotation model at the metadata level and the interface level. Our goal is to represent annotation specifications aiming at standardizing the annotation modeling to ensure an interworking between exchanging applications of the metadata. We first examined the RDF schema. The hyperlink structure of RDF schema provides flexibility and versatility to support various data formats required in collaborative multimedia annotation retrieval and exchanges. Annotea (W3C) adopted the RDF schema.

Although RDF schema is suitable for defining annotation metadata, it is not, and it was not meant to be, a general-purpose knowledge representation language. In addition, RDF does not provide a universal access approach that is critical to a Web-based annotation management system. For example, it may be neither practical nor desirable for every user to use the same annotation management system, and one may expect to have access to some annotations recorded by another annotation management system. Therefore, we investigated the Web Services Resource Framework (WSRF 2004) technology that is an XML-based presentation method to capture resources using the Web services technology.

The rapidly emerged Web services technology enables interoperability among software modules and components regardless of their underlying platforms and programming languages. A software module wrapped with a Web service interface becomes universally accessible using standard communication protocols. Particularly, WSRF provides a technique to model a universal resource with a Web services interface and access points.

We adopted WSRF to define the interface and enable interactions between annotation knowledge through Web services interfaces. Moreover, WSRF allows us to model stateful annotation knowledge, so that conversational status can be maintained and managed. Applying the WSRF technique, we model an annotation object as a universal resource. When an annotator tries to comment on an annotated document for the first time, the annotated object is identified as a resource and wrapped by a Web services interface. 


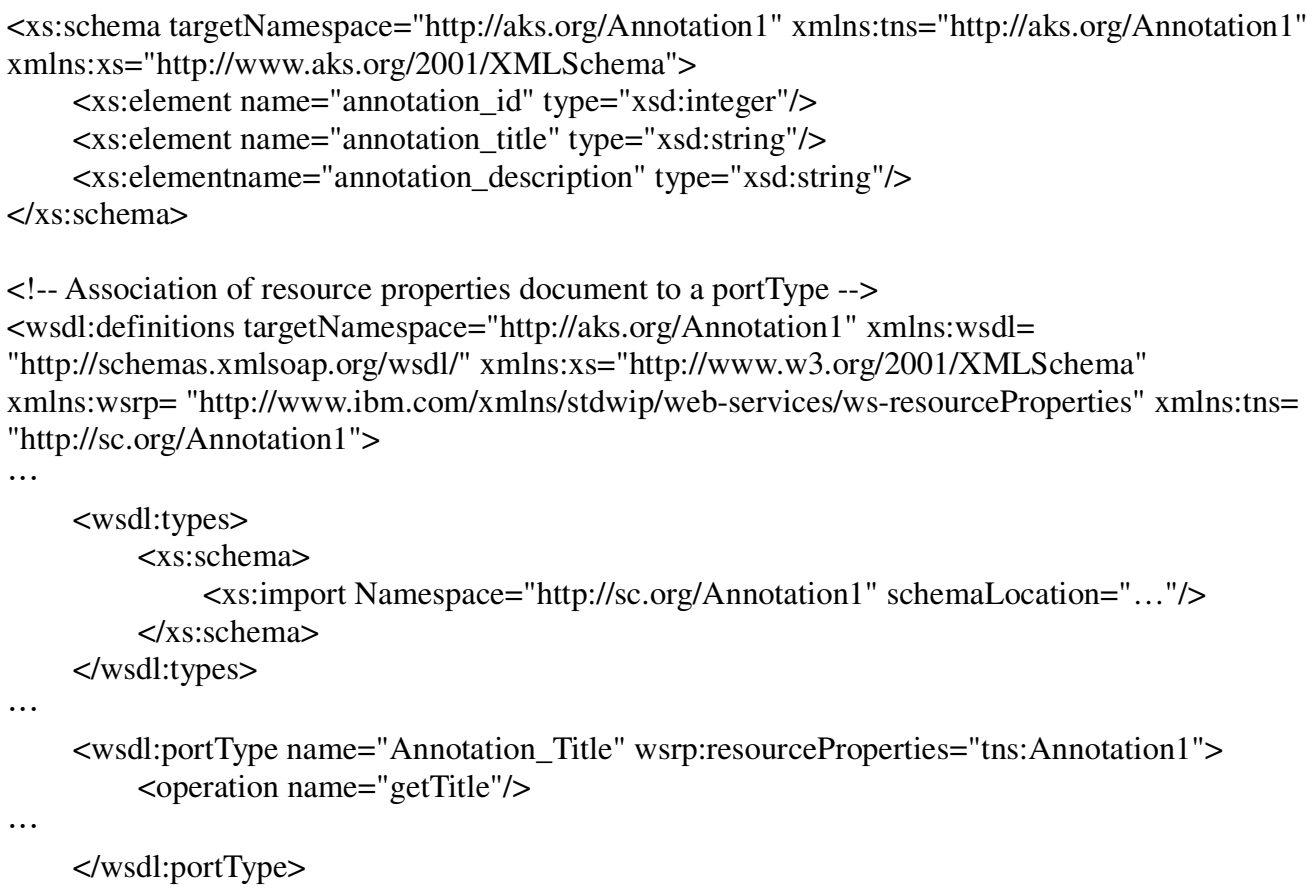

Figure. 2. A segment of an example annotation definition using WSRF.

Meanwhile, annotations are modeled as Web services-wrapped resources as well. Figure 2 shows a simplified example of resource properties document "Annotation1." The WS-Resource properties specification document is defined using XML Schema. The state of the modeling resource contains two components: annotation_id and annotation_title, with XML Schema Definition (XSD) type integer and string, respectively. In order for a user to know that the "Annotation1" defines the WS-Resource properties document associated with the Web service, the WS-Resource properties document declaration is associated with the Web Service Definition Language (WSDL) portType definition in the WSDL definition of the Web service interface, through the use of a standard attribute resourceProperties. As a result, as shown in Figure 2, the portType, with the associated resource properties document, defines the type of the WS-Resource.

Modeling annotated objects and annotations as separate WSRF resources provides several significant advantages. First, annotated documents and their annotations may be stored and managed by different groups at different locations, and integrated or bound together on an on-demand basis. Second, we enable and facilitate dynamic creation of the properties and associated values of annotation knowledge. Third, we can define a standard set of message exchanges for querying and updating the property values of annotation knowledge instances. 


\subsection{PAMS 2.0}

On the basis of our ontological metadata presentation technique, we built PAMS 2.0 as a Web-based collaborative annotation management system. For the rest of the paper, PAMS refers to PAMS version 2.0. As shown in Figure 3, PAMS 2.0 adopts a variation of the client/server architecture, whose system architecture comprises two separate parts: an annotator side and a server side. On the server side, four managers collaborate to provide four system-level supporting services: anchoring management (dynamically bind annotations to corresponding annotated sections), document management, association management, and annotator management. The technical details of implementing the four managers can be found in our previous report (Yang, Chen et al. 2004). Data recording annotations, annotated objects, annotated documents, their associations, and corresponding annotators are stored in four repositories: annotation repository, annotated document repository, association repository, and annotator repository.

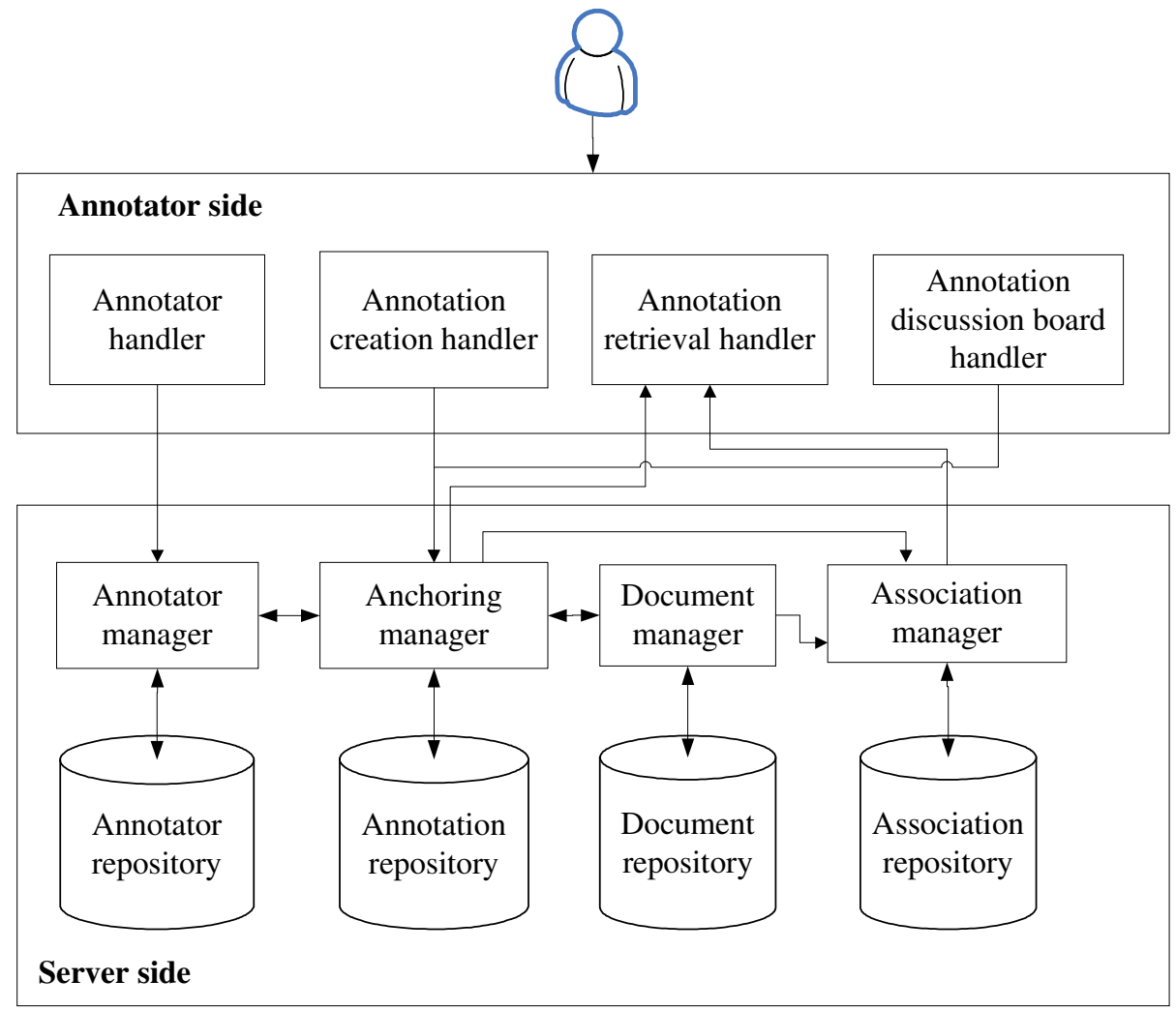

Figure 3. PAMS system architecture

On the annotator side, four handlers collaborate to process annotation creation, retrieval, discussion, and management. Annotators can create, edit, and retrieve their own annotations. In addition, annotators can retrieve annotations from other 
annotators in the same annotator group. Furthermore, annotators can discuss with each other through PAMS. Admission and access control is handled by the annotator manager, using a predefined set of roles. In the current version of PAMS, we realize a straightforward access control. An annotated document can only be freely annotated by the members from a predefined annotator group. The creator of a specific annotation may constraint that only certain roles in the annotator group have access to the annotation. New annotators may join in the group in the run time. More comprehensive and customizable access control mechanism will be our future research topic.

\section{PAMS 2.0 for knowledge sharing}

We apply our PAMS 2.0 to CSCL as a Web-based collaborative annotation tool to facilitate students in creating and sharing annotations over the Internet. Most of the collaborative annotation systems use electronic messages to inform the creation of a shared annotation. By contrast, our PAMS 2.0 supports collaboration on shared annotation using an online discussion board. Annotators can comment on, answer and/or ask questions on created annotations. This allows participating annotators to exchange their annotations directly on the annotated document.

\subsection{Online annotation for knowledge sharing}

PAMS 2.0 provides a cluster of system-level supporting services such as annotation creation, editing, and retrieval. Four categories of annotation are supported in PAMS 2.0: (1) definitions (e.g., descriptions and explanations), (2) comments (e.g., opinions and arguments), (3) questions (e.g., problems and answers), and (4) associations (e.g., additional links to other resources). Each category represents a corresponding annotation type describing certain semantic meanings of annotators' intention. PAMS 2.0 supports multiple formats of annotated documents including pdf, Microsoft Word, and HTML Web pages. An annotated object may be a word, a phrase, a sentence, an icon, an image, or a block in an annotated document. An annotator may make annotations on an annotated object in various forms such as text, image, and voice.

Figure 4 shows a screen shot of PAMS 2.0, where a user has created some annotations on a portion of the annotated document. Detailed annotation information is recorded, based on the semantic annotation definition items discussed in (Yang, Chen et al. 2004), including the location of the annotated object, the annotator, comment text content and its color, and a link to the recorded voice comment.

Figure 5 shows the Graphical User Interface (GUI) of PAMS 2.0 for an annotator to make annotations on a shared document. As shown in the screen shot, the central component of the GUI is a document browser, where an annotator can view the 
document as well as associated annotations. PAMS 2.0 integrates an input schema editor that allows annotators to specify the preferred target format such as length and type. Figure 5 also shows that annotators can attach annotation files to annotations, by specifying the location of the file in the Input Schema panel.

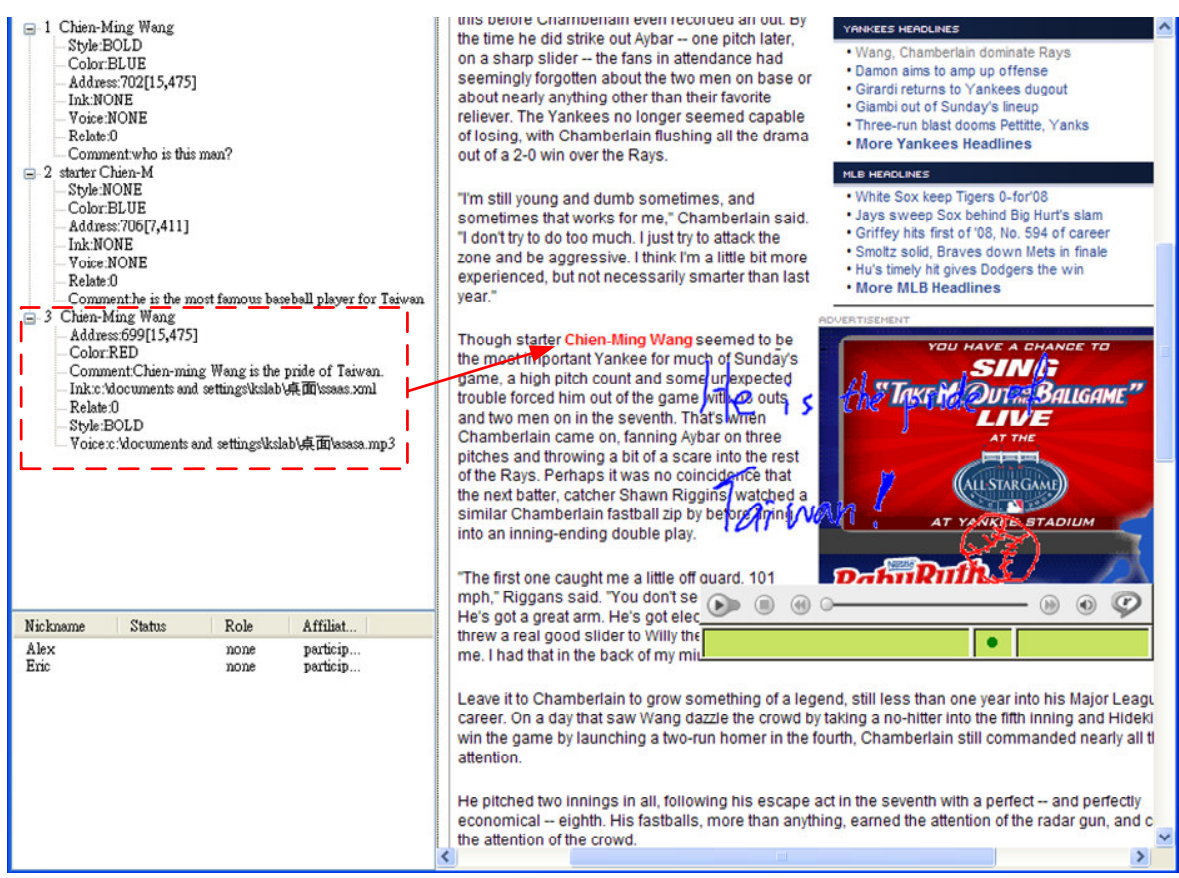

Figure 4. A screen shot of PAMS 2.0.

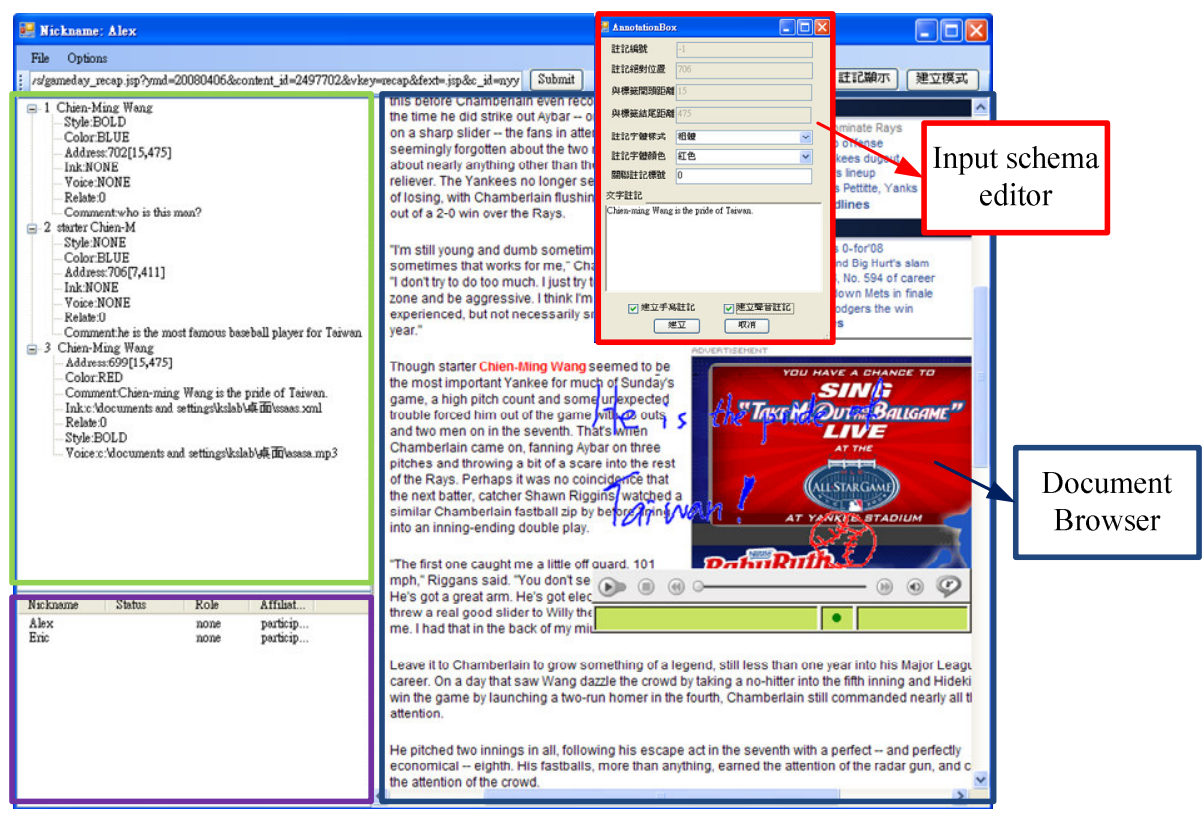

Figure 5. Graphical user interface of the annotator side in PAMS 2.0. 


\subsection{Online discussion for knowledge sharing}

Figure 6 illustrates a screen shot of how PAMS 2.0 helps annotators further share knowledge through discussing and exchanging annotations. A group user (Alex) selects an annotation document and a collaborator (Eric) to initiate a private communication channel "chat." The shared document is shown on both users' screens. One user (Alex) creates an annotation; the other user (Eric) receives the annotation; and the latter (Eric) responses to the annotation by typing some answers in the chat window. As shown in Figure 6, all annotation-related activities are recorded by PAMS 2.0 for later annotation retrieval.

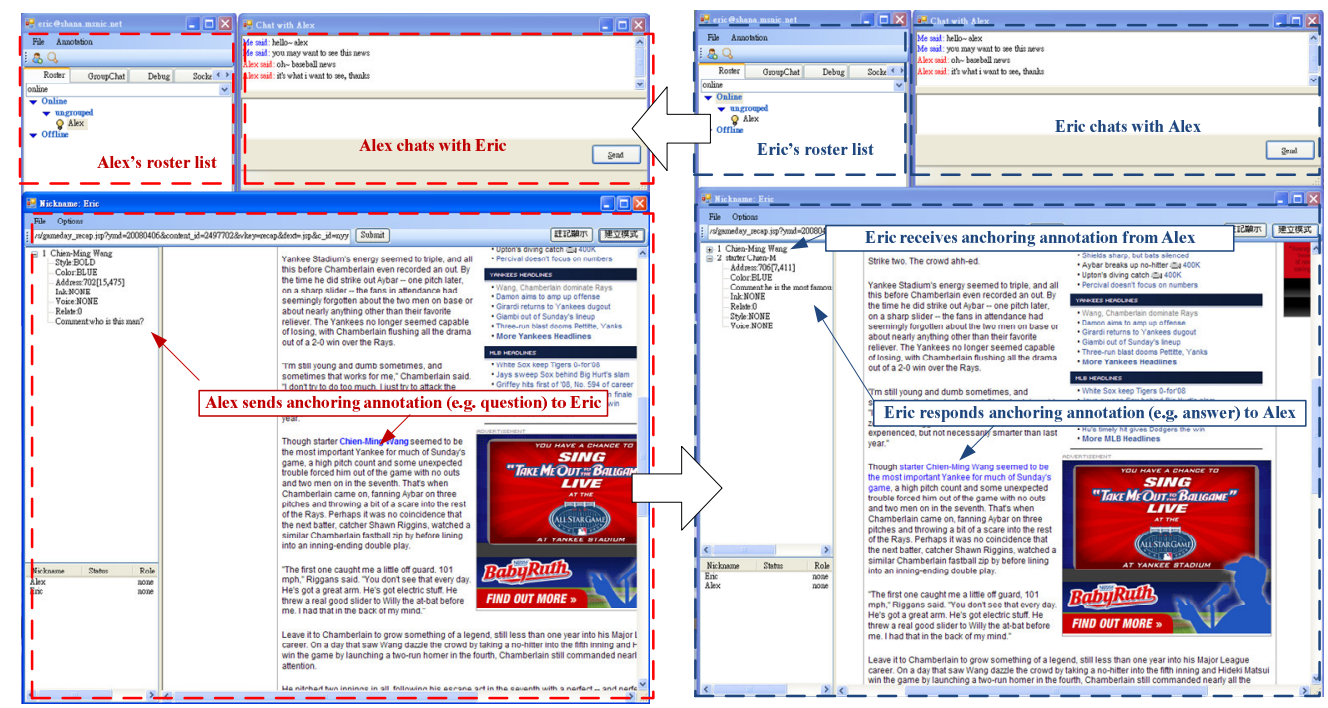

Figure 6. Students' online group reading activity with PAMS 2.0.

\section{Experiments and discussions}

We have designed and conducted a series of experiments to evaluate whether PAMS 2.0, as a Web-based annotation system, could enhance knowledge sharing in a collaborative e-learning context. Specifically, we examine whether PAMS 2.0 could help participants raise good questions and provide answers through the practice of reading, commenting, reviewing, and discussion. The rationale behind this experiment is that the efficiency of reading comprehension should be evaluated not only by tests (e.g., examinations and assessments) but also by participants' ability to raise good questions and provide proper answers (Baraka \& Rafaelib 2004).

\subsection{Experimental design}

We utilize PAMS 2.0 for students to practice online group reading and commenting, in order to enhance communication and collaboration among participants in a CSCL 
context. A group of student volunteers were randomly divided into two teams: team 1 (experiment class) that uses PAMS in their group reading activity and team 2 (counterpart class) that does not use PAMS. Each team forms a collaborative e-learning group using the corresponding platform: team 1 uses PAMS as their platform; team 2 uses other channels (emails, instant messengers, and paper documents). Tutorials and demos were given to team 1 students prior to the experiments to teach them how to utilize PAMS to read articles, annotate on the articles, find additional materials related to the articles, find other collaborators in a community of practice who are also reading the same articles, and find participants' annotations on the articles.

In one experiment, both teams were given the same reading materials. Each member in a team read, commented, raised questions, and provided answers, while discussing with other collaborators in the same team. Team members may comment on each other's annotations. At the beginning of the experiment, each team identified a team leader, who was in charge of documenting and aggregating these aforementioned collaborative events. At the end of each experiment, each team turned in a detailed team comment report, together with a set of questions raised by the team members. Accumulated questions from both teams form a question bank. We then conducted a test to evaluate participants' reading comprehension regarding the assigned materials. The examination paper was compiled by the instructor by selecting questions from the question bank provided by both teams. Every participant was required to take the examination.

The experiments were conducted at National Central University in the Spring semester of 2007. 94 junior students volunteered in the experiment. In order to ensure the two teams have similar reading ability, we required that every volunteered student take a reading test prior to the experiments. All students were sorted based on the scores they obtained from the test; then they were alternatively assigned to the two teams. 48 students were assigned to team 1 and 46 to team 2 .

Lasting for the entire semester, the experiments comprised 5 iterations, each lasting 3 weeks. In each iteration, a set of group reading materials (articles) were assigned to both teams. At the beginning of each iteration, we gave the same test to all students in both teams; at the end of each iteration, both teams took another test generated from the question bank. The reason why we adopted five iterations instead of one was three-fold. First, we tried to eliminate random issues that may affect our evaluation. Second, the students in the experiment class (use PAMS 2.0) might feel more motivated to discuss when they first used our PAMS 2.0 as a new tool, so their early test scores might not be accurate. Third, such a multiple-iteration-test assures a controlled test scenario. With five iterations being applied to the same team, we expect more reliable results since our testees remain their same reading backgrounds. In addition, in such an experimental setting, we can ensure a relatively decent number of students in every test sample (about 47 students). 


\subsection{Experimental results}

To evaluate whether PAMS helped collaborative e-learning, we compared the average scores between the two teams before and after group reading activity, as shown in Table I. For each test, the scores of all students in the same team were accumulated and an arithmetic mean was calculated. The mean values obtained from the five iterations were accumulated again to reach a final average score. In addition, for each team in every iteration, we calculated the mean deviation that is the mean of the distances between each score and the mean value. The mean deviation gave us an idea of how spread out from the average score the set of values were.

Table I lists our experimental results. The students in the experiment class achieved averagely much higher scores after group reading using PAMS (76 vs. 56). The students in the counterpart class made less progress in their scores after group reading using other methods (65 vs. 78). The mean deviation values shown in Table I indicate that our evaluation results are reliable.

Table I. Performance in group reading activity with/without PAMS.

\begin{tabular}{lcccccc}
\hline \hline \multicolumn{7}{c}{ Experiment Class (48 students) } \\
\hline Iteration \# & 1 & 2 & 3 & 4 & 5 & Average \\
\hline Ave. Scores (before) & 53 & 54 & 58 & 56 & 59 & 56 \\
Mean deviation & $(8.58)$ & $(8.69)$ & $(7.25)$ & $(6.01)$ & $(5.58)$ & \\
\hline Ave. Scores (after) & 74 & 72 & 77 & 77 & 80 & 76 \\
Mean deviation & $(7.85)$ & $(6.98)$ & $(6.51)$ & $(6.19)$ & $(7.12)$ & \\
\hline & Counterpart Class (46 students) & & \\
\hline Iteration \# & 1 & 2 & 3 & 4 & 5 & Average \\
\hline Ave. Scores (before) & 53 & 56 & 55 & 61 & 65 & 58 \\
Mean deviation & $(8.31)$ & $(7.56)$ & $(6.93)$ & $(5.11)$ & $(4.66)$ & \\
\hline Ave. Scores (after) & 65 & 59 & 60 & 68 & 73 & 65 \\
Mean deviation & $(7.52)$ & $(5.41)$ & $(6.15)$ & $(7.02)$ & $(6.54)$ & \\
\hline
\end{tabular}

To better illustrate our experimental findings, the evaluation results are summarized in Figure 7. Before group reading, the average scores of both teams are similar. After group reading activity, team 1 using PAMS significantly exceeds team 2 in material understanding. This experimental result demonstrates that collaborative annotation helps to improve students' reading comprehension in a collaborative e-learning context. 


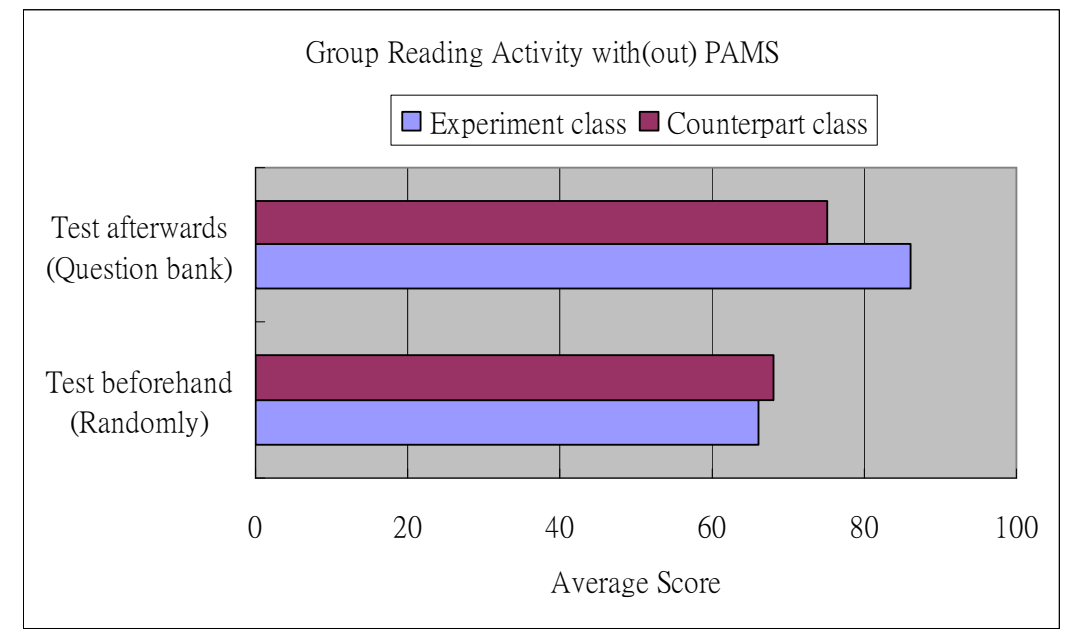

Figure 7. Examination performance of experiment group vs. counterpart group.

We also designed a questionnaire with a list of yes/no questions for the students in team 1 at the end of the experiments, for examining whether they prefer using PAMS for group e-learning and the aspects they like it. The answers were positive, and the top three reasons why the students like to use PAMS are its capability of helping students to (1) comment, (2) discuss, and (3) raise question-answers.

Through the survey, the students confirmed that in addition to enhancing their thinking skills by practicing personalized annotations, PAMS encourages their attitudes toward knowledge sharing. The students also confirmed that by making annotations and discussing with other annotators in the study group through related annotations, PAMS facilitates knowledge sharing.

We further studied the data from team 1 by examining the relationships between individual students' examination performance and the number of questions raised by them as well as the number of questions adopted in the examination paper. As shown in Figure 8 , by using PAMS, students' examination performance improved when they raised more questions. This indicates that the more questions students raise, the more actively they involve in knowledge sharing, and the better their examination performance.

In summary, we found that a Web-based annotation system can be applied to the collaborative e-learning field to foster knowledge sharing by enhancing interactive communication and collaboration among participating students. 

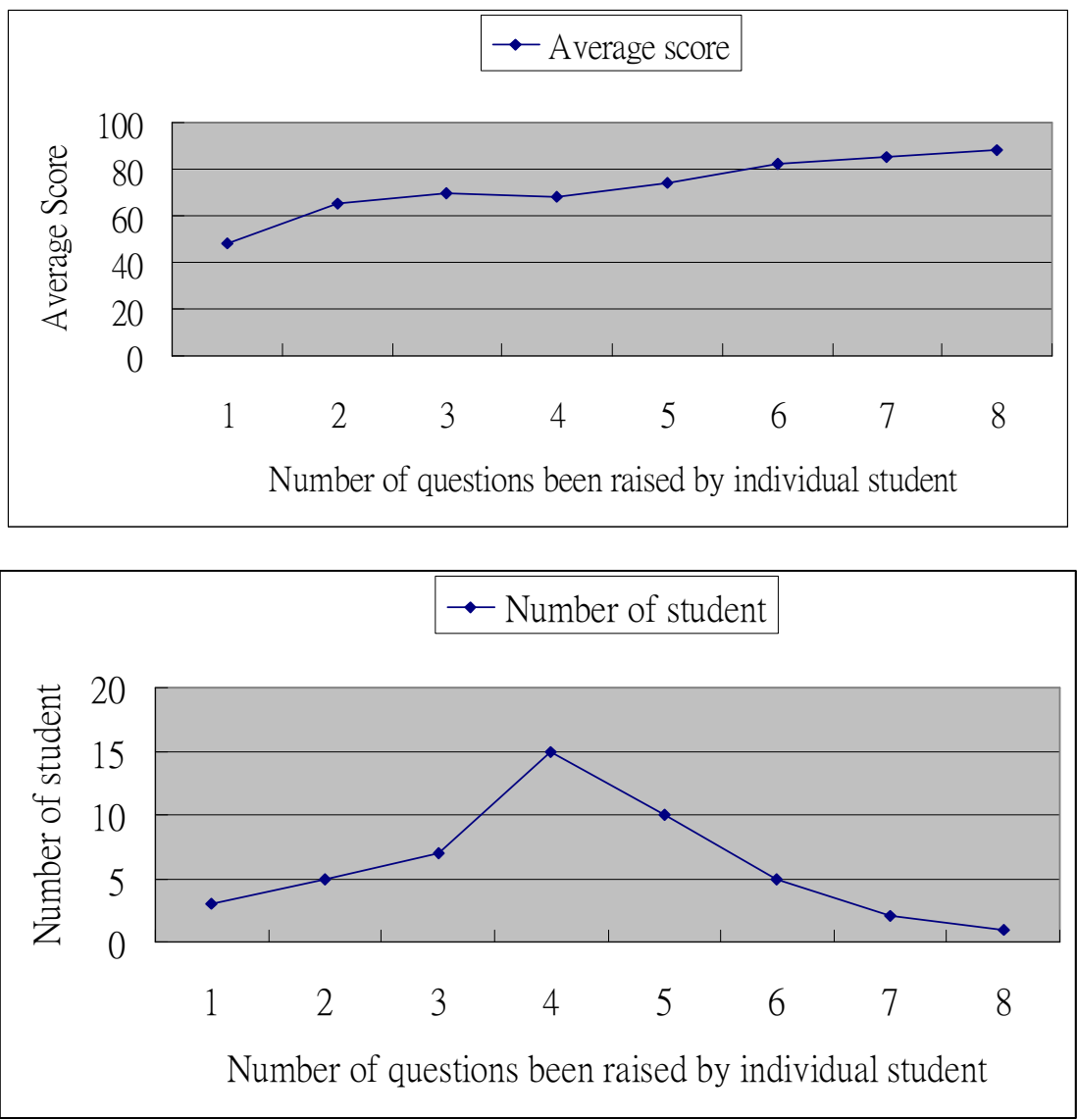

Figure 8. Students' examination performance vs. the number of questions they raised.

\section{Conclusions}

Computer supported collaborative learning (CSCL) has become an important way to enhance learning and teaching. The behaviors of making marks on reading documents are important in CSCL. Multimedia annotation-based collaboration can improve the process of reading as well as student's comprehension of learning materials. In this paper, we have presented our personalized annotation management system (PAMS) 2.0 as a Web-based social software tool for collaborative e-learning. Our study demonstrates that collaborative multimedia annotation enhances knowledge sharing in students' online group reading activity. Our research also demonstrates that the Semantic Web and Web services technologies can be applied to enhance interoperability and maintainability of collaborative e-learning software.

We expect that PAMS can be extended and commercialized to support collaborative e-learning and knowledge sharing in two ways: either as an integral component to plug into an online collaboration platform or as an independent Web service to serve as an underlying collaboration platform. Furthermore, we expect that 
PAMS can be extended to support a broad sense of Internet-based communication and collaboration in a context of a community of practice, not limited to e-learning but expanded to business and social interactions.

In the current version of PAMS, our role-based admission and access control is straightforward and rather simple. We plan to construct more comprehensive role-based access control facility allowing collaborators to dynamic configure and reconfigure access control rules. We also plan to construct a dedicated Web site to host our PAMS that is universally accessible to all Internet users. In addition, we plan to explore the feasibility and performance of using PAMS (including annotation creation and browsing) through various mobile devices (e.g., PDAs and mobile phones). We plan to mobilize the discussion facility of PAMS and to make the annotation retrieval more context-aware and social-aware.

\section{Acknowledgements}

This work is supported by National Science Council, Taiwan under grants NSC95-2520-S-008-006-MY3 and NSC96-2628-S-008-008-MY3.

\section{References}

E.G. Aiken, G.S. Thomas and W. Shennum (1975). "Memory For A Lecture: Effect Of Notes, Lecture Rate, and Information Density." Journal of Education Psychology: 439-444.

H. Alani, S. Dasmahapatra, K. O'Hara and N. Shadbolt (2003). "Identifying Communities of Practice through Ontology Network Analysis." IEEE Intelligent Systems. 18(2): 18-25. N. Avouris, V. Komis, M. Margaritis and G. Fiotakis (2004). "An environment for studying collaborative learning activities." Educational Technology \& Society. 7(2): 34-41.

M. Baraka and S. Rafaelib (2004). "On-line Question-Posing and Peer-Assessment as Means for Web-Based Knowledge Sharing in Learning." Human-Computer Studies. 61: 84-103. C. Bravo, M.A. Redondo, M. Ortega and M.F. Verdejo (2006). "Collaborative environments for the learning of design: a model and a case study in Domotics." Computers \& Education. 46(2): 152-173.

D. Brickley. (2004). "Collaboration, knowledge representation and automatability." Retrieved May, 21, 2008, From the Internet Poetry Archive web site: http://www.w3.org/Collaboration/.

S.A. Carter, E.F. Churchill, L. Denoue, J.I. Helfman and L. Nelson (2004). Digital graffiti: public annotation of multimedia content. International Conference for Human-Computer Interaction, 1207-1210.

S. Chung, C. Severance and M.-J. Chung (2003). "Design of support tools for knowledge building in a virtual university course." Journal of Interactive Learning Environments. 11(1): 41 $-57$.

F. Fischer, J. Bruhn, C. Gräsel and H. Mandl (2002). "Fostering Collaborative Knowledge Construction with Visualization Tools." Learning and Instruction. 12: 213-232.

D. Gašević, J. Jovanović and V. Devedžić (2007). "Ontology-based annotation of learning object content." Journal of Interactive Learning Environments. 15(1): 1-26.

K. Guldberg and R. Pilkington (2006). "A Community of Practice Approach to the Development of Non-Traditional Learners through Networked Learning." Journal of Computer Assisted Learning. 22: 159-171.

W.Y. Hwang, C.Y. Wang and M. Sharples (2007). "A study of multimedia annotation of Web-based materials." Computer \& Education. 48(4): 680-699.

IBM. "Lotus Notes." from http://www-01.ibm.com/software/lotus/. 
T.D.C.M. Initiative "Dublin Core."

V. Kollias, N. Mamalougos, X. Vamvakoussi, M. Lakkala and S. Vosniadou (2005). "Teachers' Attitudes to and Beliefs about Web-Based Collaborative Learning Environments in the Context of An International Implementation." Computers \& Education. 45: 295-315.

V. Komis, N. Avouris and C. Fidas (2002). "Computer-Supported Collaborative Concept Mapping: Study of Synchronous Peer Interaction." Education and Information Technologies. 7(2): 169-188.

T. Koschmann (1996). CSCL: Theory and Practice of an Emerging Paradigm (Computers, Cognition, and Work), Lawrence Erlbaum Associates Inc., Mahwah, NJ, USA.

K. Kreijns, P.A. Kirschner, W. Jochems and H.v. Buuren (2005). "Measuring Perceived Sociability of Computer-Supported Collaborative Learning Environments." Computers \& Education: (in press).

K. Kreijns, P.A. Kirschner, W. Jochems and H.v. Buuren (2007). "Measuring Perceived Sociability of Computer-Supported Collaborative Learning Environments." Computers \& Education. 49(2): 176-192.

K. Littleton and P. Häkkinen (1999). Learning Together: Understanding the Processes of Computer-Based Collaborative Learning. Collaborative Learning: Cognitive and Computational Approaches (ed. P. Dillenbourg), Elsevier Science, London, UK: 20-30.

LOM "IEEE WG 12: Learning Object Metadata."

J. Lundin and M. Magnusson (2003). "Collaborative learning in mobile work." Journal of Computer Assisted Learning. 19(3): 273-283.

S. Manlove, A.W. Lazonder and T. Jong (2006). "Regulative Support for Collaborative Scientific Inquiry Learning." Journal of Computer Assisted Learning. 12: 87-98.

Microsoft. (2007). "OneNote." from http://office.microsoft.com/en-us/onenote/default.aspx.

D.J. Nicol and I.A. MacLeod (2005). "Using a shared workspace and wireless laptops to improve collaborative project learning in an engineering design class." Computers \& Education. 44(4): 459-475.

W. Rubens, B. Emans, T. Leinonen, A.G. Skarmeta and R.J. Simons (2005). "Design of web-based collaborative learning environments: translating the pedagogical learning principles to human computer interface." Computers \& Education. 45(3): 276-294.

B. Russell (2005). "Annotation." The collected papers of bertrand russell. 1(12): 451-568. T. Sannomiva, T. Amagasa, M. Yokishawa and S. Uemura (2001). A Framework for Sharing Personal Annotations on Web Resources Using XML. the 2001 Workshop on Information Technology and Virtual Enterprises, 40-48.

SCORM "Advanced Distributed Learning Network Shareable Content Object Reference Model."

M. Surman and D. Wershler-Henr (2002). Commonspace: Beyond Virtual Community, Prentice Hall PTR.

W3C. "Amaya." from Retrieved 2008, From the Internet Poetry Archive web site:

http://www.w3.org/Amayal.

W3C "Annotea."

E. Wenger, R. McDermott and W. Snyder (2002). Cultivating Communities of Practice: A Guide to Managing Knowledge, Harvard Business School Press, Boston, MA, USA.

WSRF. (2004). "Web Services Notification and Web Services Resource Framework (WSRF)." from http://www-106.ibm.com/developerworks/webservices/library/ws-resource/.

S. Yang, J. Zhang and I. Chen (2007). Web 2.0 Services for Identifying Communities of Practice through Social Networks. IEEE International Conference on Services Computing (SCC 2007), Salt Lake City, UT, USA, Jul. 9-13, 130-137.

S.J.H. Yang, I. Chen and N. Shao (2004). "Ontological Enabled Annotations and Knowledge Management for Collaborative Learning in Virtual Learning Community." Journal of Educational Technology and Society. 7(4): 70-81.

S.J.H. Yang and I.Y.L. Chen (2008). "A social network-based system for supporting interactive collaboration in knowledge sharing over peer-to-peer network." International Journal of Human-Computer Studies. 66(1): 36-50.

J. Zumbach, A. Hillers and P. Reimann (2003). "Supporting distributed problem-based learning: the use of feedback in online learning." Online Collaborative Learning: Theory and Practice: 86-103.

G. Zurita and M. Nussbaum (2004a). "Computer Supported Collaborative Learning Using 
Wirelessly Interconnected Handheld Computers." Computers \& Education. 42: 289-314. G. Zurita and M. Nussbaum (2004b). "Computer Supported Collaborative Learning using Wirelessly Interconnected Handheld Computers." Computers \& Education. 42: 289-314. 